\title{
Motor coordination or balance degradation during microwave energy exposure
}

\author{
ALLAN H. FREY and SHIELA GENDLEMAN \\ Randomline, Inc., Huntingdon Valley, Pennsylvania 19006
}

\begin{abstract}
The effect of pulse-modulated microwave energy on motor coordination or balance was evaluated. A horizontal rod, on which a rat balanced, rotated at an increasing speed until the rat fell off. The Sprague-Dawley rats were divided into two groups: sham exposed and microwave energy exposed. The microwave energy exposure occurred during their time on the rotating rod. The time scores of the animals on the rod were recorded, and it was found that the microwave energy-exposed animals were adversely affected.
\end{abstract}

In recent years, microwave energy has become a pervasive and increasingly significant factor in our environment. In this decade, a series of experiments have shown diverse behavioral effects of exposure to low-intensity microwave energy. It is becoming clear that the primary influence of the energy is on the nervous system and behavior.

It has been found, for example, that humans perceive pulse-modulated energy as a sound (Frey, 1971; Frey \& Messenger, 1973). Shuttlebox behavior is influenced by the energy, with mammals avoiding exposure (Frey $\&$ Feld, 1975). Hjersen and Phillips (Note 1) also found that exposure to microwave energy influenced behavior in a shuttlebox. Gage (Note 2), using rats who were trained to alternately press two bars in an operant conditioning task, suppressed subjects' behavior by exposure to microwave energy. Gillard, Servantie, Bertharion, Servantie, Obrenovitch, and Perrin (1976) found that exploratory activity was modified by exposure to the energy. Monahan and Henton (1977) showed that microwave energy exposure can maintain an active instrumental avoidance or escape response. Mitchell, Switzer, and Bronaugh (Note 3) used microwave energy to study innate and learned behavior in rats. They found exposed animals showed statistically significant increases in locomotor activity and disrupted differential responding during asymptotic discriminative operant reward conditioning. Thomas, Yeandle, and Burch (1976) determined that rats performing on a reinforcement schedule regulated by internal stimulus control could be influenced by exposure to microwave energy. They found that energy exposure increased the frequency of premature switching.

If microwave energy exposure also affects motor coordination or balance, then such an effect would have to be considered in the interpretation of the foregoing and additional behavioral experiments. Such motor coordination or balance effects are suggested by the results of Frey and Coren (1979), who established that the microwave hearing effect is apparently due to an effect within the cochlea. In addition, Lebovitz and
Seaman (Note 4) found, through use of single-neuron recording, that vestibular units were influenced by microwave energy exposure. Further, Frey and Spector (Note 5) have proposed and provided data indicating that the dopamine systems of the brain are involved in the mechanism of microwave energy effects. The latter implies that the nigrostriatal tract, which is critical in motor coordination, may be influenced. Thus, there is reason to test the possibility of motor coordination or balance effects of microwave energy exposure. The following is a report on the results of a test of that possibility, using brief exposures to low-intensity microwave energy.

\section{GENERAL METHOD}

A horizontal rotating rod apparatus was developed for rats to balance on during exposure to microwave energy. The apparatus, shown in Figure 1, consisted of a rod, motor, and $\mathrm{U}$ frame. The rod was a 3.2-cm-diam wooden dowel, held in bearing rings mounted in a wooden $U$ frame. A variable-speed gearmotor, located behind an energy-absorbing block, drove the rod.

Two $30 \times 23 \times .65 \mathrm{~cm}$ plates of white polystyrene were

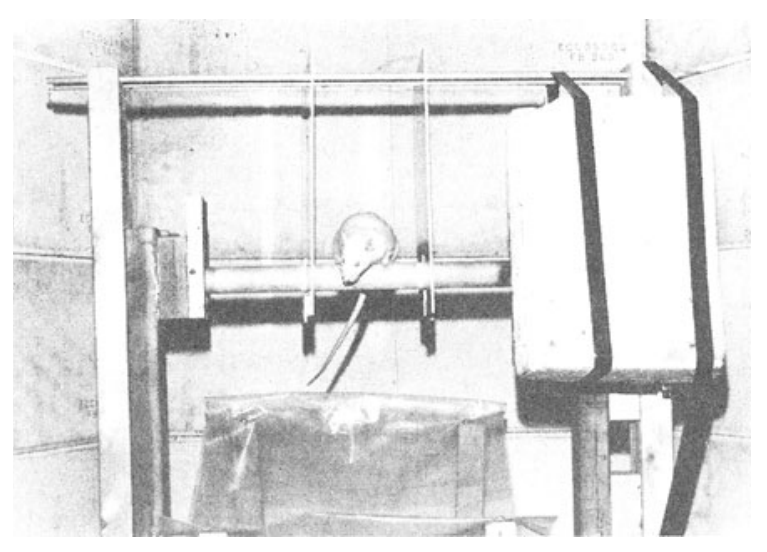

Figure 1. Rotating rod apparatus within microwave anechoic enclosure as seen from aperture of horn antenna. The animal is in the typical position. The motor is shielded by a block of microwave energy-absorbing material. 
suspended from two wooden dowels above the rod and divided the space on the rod into three sections. The two plates were notched at the bottom to fit over the rotating rod. These plates prevented the animal from walking off the end of the rod. A clear sheet of vinyl was suspended, hammock style, on a wooden frame under the rotating rod to catch the subjects as they fell from the rod. The starting speed of the rod was $14 \mathrm{rpm}$, with a maximum speed of $42 \mathrm{rpm}$ after the system was in operation for $90 \mathrm{sec}$. At the end of $2 \mathrm{~min}$ or when the animal fell off the rod, the motor control was reset.

The microwave energy was directed toward the animal located within a microwave energy anechoic enclosure. The view in Figure 1 is from the location of the horn antenna. The energy was generated by a Microdot Model 411A microwave energy generator connected by RG-8 coaxial cable to a standard gain horn antenna. The microwave frequencies used in this experimentation were $1,1.3$, and $1.5 \mathrm{GHz}$. The pulse repetition rate was $1,000 \mathrm{pps}$, with a pulse width of $.5 \mathrm{msec}$. Incident energy was measured with a dipole antenna before and after each session in the location occupied by the animal. The dipole was connected via RG-58 cable to a Microlab AF-20 attenuator located outside the chamber. The attenuator was connected to a Hewlett-Packard Model 477B thermistor mount, which connected to a Hewlett-Packard Model 430C power meter. Pilot studies suggested that threshold energy value for effect would be about $1 \mathrm{~mW} / \mathrm{cm}^{2}$. Since we wished to define threshold for small groups, the measured average incident power density used in Experiment 1 was $1.4 \mathrm{~mW} / \mathrm{cm}^{2}$, with a peak power of $2.8 \mathrm{~mW} / \mathrm{cm}^{2}$. In Experiment 2 the measured average incident power used was $.2 \mathrm{~mW} / \mathrm{cm}^{2}$, with a peak power of $.4 \mathrm{~mW} / \mathrm{cm}^{2}$.

The procedure was double blind, with one experimenter controlling the energy-exposure condition and another experimenter placing the animal on the rod and measuring its time on the rod. The animal was placed on the rod, the energy condition was put into effect, and rotation was started. Energy exposure and timing continued until the subject lost its balance. Loss of balance was defined as falling from the rod or loss of footing on the rod (e.g., animal wrapped its body around the rod). The time on the rod was recorded in seconds, with a time limit of $120 \mathrm{sec}$. All conditions of testing exposed and sham-exposed animals were the same, except for the presence or absence of the microwave energy.

\section{EXPERIMENT 1}

\section{Method}

The animals were tested over a 2-week period in six sessions. They were tested five times per session in 120-sec runs. There was an experimental and a control group for each frequency, with six animals in each group.

\section{Results}

The mean time on the rod is shown in Figure 2 for each frequency. There were significant differences between the experimental group and the control group means at 1.3 and $1.5 \mathrm{GHz}$, as shown by the MannWhitney $\mathrm{U}$ test. There was significantly less variance in the exposed groups at the $1.3-\mathrm{GHz}$ and the $1.5-\mathrm{GHz}$ frequencies, compared with the sham-exposed groups, as shown in Figure 3.

At the end of the experiment, the rotation of the rod was reversed. The animals were placed on the rod so that they were facing into the rotation as before, but not facing the antenna as before. The microwave energyexposed animals' behavior was disturbed, and their performance became erratic. Five sessions were required before their performance began to stabilize. In contrast,

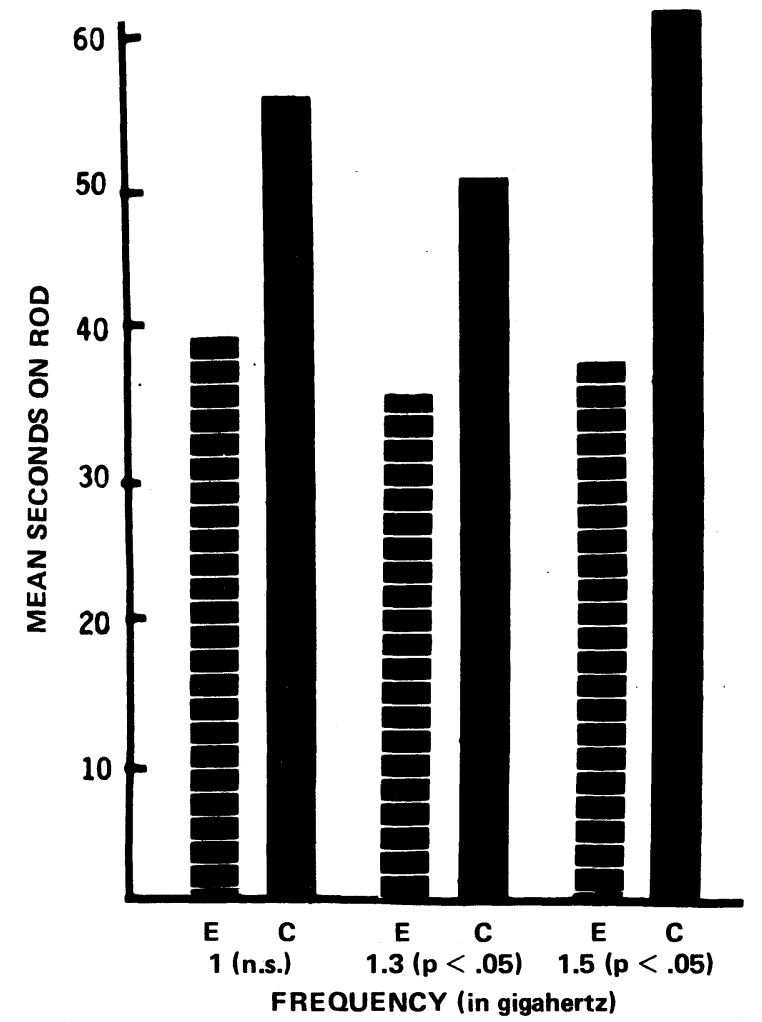

Figure 2. Mean time on the rotating rod by frequency of microwave energy used. The significance (Mann-Whitney $U$ test) for each comparison of means is shown.



Figure 3. Differences between mean variances of the groups (in relative units to equate controls for clarity in the figure). 
the animals that were sham exposed readily adapted to the change and, within the second session, were performing as previously.

\section{EXPERIMENT 2}

\section{Method}

In this experiment, new groups of animals were used. They were used in an own-control design, with half exposed to the energy first and half sham exposed first. Exposure conditions were then reversed, after 1 week of no experimentation to allow possible residual effects to dissipate. This design was used because pilot testing suggested that the power densities to be used in this experiment might be just below threshold, and an effect on variance had been shown in Experiment 1. In this factorial design, the animals were separated into two groups that were matched according to their ability on the rod during pretest trials. Animals were randomly assigned within groups to the three microwave frequencies. Group $A$ was exposed for the first half of the experiment and Group B was sham exposed, with reversal in the second half of the experiment. Testing consisted of 12 sessions, with a 1 -week break at the halfway point. Each animal was given four trials on the rotating rod during each session.

\section{Results}

There were no significant differences between experimental and control means, nor were there significant differences in the variances. The trends suggest that there was a minimal effect that could possibly be teased out by using large groups.

\section{DISCUSSION}

Pulse-modulated microwave energy with the characteristics used in this experiment has an adverse effect on motor coordination or balance as defined by the rotating rod test. The effect occurs promptly, is substantial, since it is significant with small groups, and occurs at low incident power densities in the range of incident energies frequently used in behavioral experiments with microwaves. Thus, this effect must be considered in the interpretation of many behavioral experiments involving the use of microwave energy.

The effect is also of consequence in itself. It may possibly indicate an influence of microwave energy on the nigrostriatal system of the brain, or it may indicate a labyrinthine effect. A way to separate the two systems for test with an animal in a microwave field needs to be developed.

The result of reversing rotation of the rod in Experiment 1 might be due to an altered perception of the energy or an altered nonperceived effect of the energy.

\section{REFERENCE NOTES}

1. Hjersen, D. L., \& Phillips, R. D. Perception and response to pulsed microwave radiation by rats. Paper presented at the International Union of Radio Science Annual Meeting, Amherst, Mass., October 1976.

2. Gage, M. I. Effects of single exposures to 2,450-MHz microwave irradiation on rat behavior. Paper presented at the International Union of Radio Science Annual Meeting, Amherst, Mass., October 1976.

3. Mitchell, D. S., Switzer, W. G., \& Bronaugh, E. L. Effects of chronic athermal microwave radiation on innate and learned behavior in rats. Paper presented at the International Union of Radio Science Annual Meeting, Amherst, Mass., October 1976.

4. Lebovitz, R. M., \& Seaman, R. L. Eigth nerve derived, singleneuron responses to acute microwave radiation of the head. Paper presented at the 1977 International Symposium on the Biological Effects of Electromagnetic Waves, Airlie, Va., October 1977.

5. Frey, A. H., \& Spector, J. Irritability and aggression in mammals as affected by exposure to electromagnetic energy. Paper presented at the International Union of Radio Science Annual Meeting, Amherst, Mass., October 1976.

\section{REFERENCES}

Frey, A. H. Biological function as influenced by low-power modulated RF energy. IEEE Transactions Microwave Theory and Techniques, 1971, MTT-19,2, 153-164.

Frey, A. H., \& Coren, E. Holographic assessment of a hypothesized microwave hearing mechanism. Science, 1979, 206, 232-234.

FreY, A. H., \& Feld, S. R. Avoidance by rats of illumination with low power nonionizing electromagnetic energy. Journal of Comparative and Physiological Psychology, 1975, 89, 183-188.

Frey, A. H., \& Messenger, R. Human perception of illumination with pulsed ultrahigh frequency electromagnetic energy. Science, 1973, 181, 356-358.

Gillard, J., Servantie, B., Bertharion, G., Servantie, A. M., Obrenovitch, J., \& Perrin, J. C. Study of the microwave-induced perturbations of the behavior by the open field test into the white rat. In C. C. Johnson \& M. L. Shore (Eds.), Biological effects of electromagnetic waves (Vol. 1) (HEW Publication FDA 77-8010). Washington, D.C: U.S. Government Printing Office, 1976.

Monohan, J. C., \& Henton, W. W. Free-operant avoidance and escape from microwave radiation. In D. G. Hazzard (Ed.), Proceedings of symposium on biological effects and measurement of radio frequency.'microwaves (HEW Publication FDA 778026). Washington, D.C: U.S. Government Printing Office, 1977.

Thomas, J. R., Yeandle, S. S., \& Burch. Modification of internal discriminative stimulus control of behavior by low levels of pulsed microwave radiation. In C. C. Johnson \& M. L. Shore (Eds.), Biological effects of electromagnetic waves (Vol. 1) (HEW Publication FDA 77-8010). Washington, D.C: U.S. Government Printing Office, 1976.
(Received for publication October 22, 1979.) 